\title{
Primer registro de Unguiculariopsis ravenelii (Leotiomycetes, Ascomycota) en México
}

\author{
First record of Unguiculariopsis ravenelii \\ (Leotiomycetes, Ascomycota) from Mexico
}

Acta Botanica Mexicana

\author{
Tania Raymundo' (D), Michelle Martínez-Pineda' (D), Aurora Cobos-Villagrán' (D), Marcos Sánchez-Flores' (D) y Ricardo \\ Valenzuela!,2 (D)
}

\section{Resumen:}

Antecedentes y Objetivos: El género Unguiculariopsis de la clase Leotiomycetes dentro de Ascomycota se caracteriza por formar apotecios inconspicuos de hasta $2 \mathrm{~mm}$ de diámetro, con una superficie formada por pelos en forma de gancho, ascas inoperculadas, inamiloides y ascosporas globosas, subglobosas a elipsoidales, hialinas con gútulas. Son hongos fungícolas que presentan especificidad por el hospedero; en el presente estudio se tiene por objetivo reportar a Unguiculariopsis ravenelii por primera vez en México.

Métodos: Los especímenes estudiados están depositados en el herbario ENCB del Instituto Politécnico Nacional, México. Los materiales fueron revisados en fresco y en seco, se describieron macro morfológicamente usando un estereoscopio, fotografías tomadas in situ y micro morfológicamente con un microscopio óptico. Se identificaron con claves y literatura especializada.

Resultados clave: Se presenta la descripción de Unguiculariopsis ravenelii de México, una especie que se caracteriza por ser micoparásita y por formar apotecios gregarios sobre Rhytidhysteron rufulum.

Conclusiones: Esta especie se distribuye en bosque tropical caducifolio y produce sus esporomas durante la temporada de lluvias en los estados de Colima, Jalisco, Nayarit, Oaxaca, Puebla, Sonora, Tabasco y Tamaulipas en la región Neotropical de México.

Palabras clave: Cordieritidaceae, Cyttariales, fungícola, micoparásito, Rhytidhysteron rufulum.

\section{Abstract:}

Background and Aims: The genus Unguiculariopsis of the class Leotimycetes within Ascomycota is characterized by forming inconspicuous apothecia up to $2 \mathrm{~mm}$ in diameter, with hook-shaped hairs on the external surface, inoperculate and inamyloid asci, and globose, subglobose to ellipsoid ascospores, which are hyaline with guttules. They are fungicolous fungi which are host-specific. The objective of this study is to report Unguiculariopsis ravenelii for the first time in Mexico.

Methods: The specimens studied are deposited in the ENCB herbarium of the Instituto Politécnico Nacional, Mexico. The material was reviewed in fresh and dry, and described macromorphologically using a stereomicroscope, based on pictures taken in situ, and micromorphological characters with an optical microscope. They were identified with specialized keys and literature.

Key results: The description of Unguiculariopsis ravenelii of Mexico is presented. This is a species characterized by being mycoparasite and forming gregarious apothecia growing on Rhytidhysteron rufulum.

Conclusions: This species is distributed in tropical dry forest and produces its sporomas during the rainy season in the states of Colima, Jalisco, Nayarit, Oaxaca, Puebla, Sonora, Tabasco and Tamaulipas in the Neotropical region of Mexico.

Key words: Cordieritidaceae, Cyttariales, fungicolous, mycoparasite, Rhytidhysteron rufulum.

${ }^{1}$ Instituto Politécnico Nacional, Escuela Nacional de Ciencias Biológicas, Departamento de Botánica, Laboratorio de Micología, 11340 Cd. Mx., México.

²Autor para la correspondencia: rvalenzg@ipn.mx
Recibido: 30 de enero de 2020.

Revisado: 21 de febrero de 2020.

Aceptado por Marie-Stéphanie Samain: 23 de marzo de 2020.

Publicado Primero en línea: 15 de abril de 2020.

Publicado: Acta Botanica Mexicana 127 (2020).
Citar como:

Raymundo, T., M. Martínez-Pineda, A. Cobos-Villagrán, M. Sánchez-Flores y R. Valenzuela. 2020. Primer registro de Unguiculariopsis ravenelii (Leotiomycetes, Ascomycota) en México. Acta Botanica Mexicana 127: e1666. DOI: 10.10.2189/abm127.2020.1666

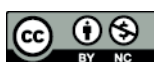

Este es un artículo de acceso abierto bajo la licencia Creative Commons 4.0 Atribución-No Comercial (CC BY-NC 4.0 Internacional).
e-ISSN: 2448-7589 


\section{Introducción}

El género Unguiculariopsis Rehm pertenece a la famila Cordieritidaceae, orden Cyttariales, clase Leotiomycetes, Phylum Ascomycota (Ekanayaka et al., 2019). Está conformado por 31 especies reconocidas (Index Fungorum, 2020), forma fructificaciones inconspicuas, que se caracterizan por el desarrollo de apotecios gregarios con el margen enrollado, himenio cinabrio a marrón vináceo en seco; con la superficie externa blanquecina a grisácea-furfurácea, cubierta por pelos de ápice ganchudo, ligeramente marrón en la base con el ápice hialino; excípulo ectal de textura globosa a angularis; paráfisis filiformes, ligeramente a fuertemente deformadas en el ápice, hialinas, con o sin septos; ascas inoperculadas, octospóricas, cilíndricas, subcilíndricas, clavadas o subfusoides con al ápice inamiloide; ascosporas uni o biseridas, globosas, subglobosas o elipsoidales, hialinas a pálido amarillentas, unicelulares con una gútula de paredes lisas. El hábito de estos hongos se ha determinado como fungícola y liquenícola. No obstante, Zhuang $(1988,2000)$ menciona que el término adecuado es micoparásito y que además presentan especificidad por el hospedero, causa por la cual las ascosporas no germinan en medio artificial. Hawksworth (1993) señaló que la forma adecuada de indicarlos es biótrofos hiperparásitos. El género es cosmopolita (Hairaud, 2014) y presenta 31 especies válidas (Index Fungorum, 2020). Una de las más frecuentes es Unguiculariopsis ravenelii (Berk. \& M.A. Curtis) W.Y. Zhuang \& Korf, la cual fue descrita como Cenagiun ravenelii (Berk. \& Br.) Sacc., creciendo sobre Patellaria nigro-cinnabarina Schwein. (=Rhytidhysteron rufulum (Spreng.) Speg.) (Korf, 1971). Esta especie ha sido ampliamente citada de Austria, Brasil, China, Colombia, Estados Unidos de América, Francia, Jamaica, Japón, Perú, Surinam y Venezuela (Zhuang, 1988; GBIF, 2020). El presente estudio tiene como objetivo reportar a Unguiculariopsis ravenelii por primera vez para México.

\section{Materiales y Métodos}

Se revisó la colección de hongos del Herbario de la Escuela Nacional de Ciencias Biológicas (ENCB) del Instituto Politécnico Nacional (IPN). A los especímenes se les realizaron cortes transverso-longitudinales de los apotecios, con na- vajas de doble filo y se hicieron preparaciones temporales en alcohol a $70 \%$ y $\mathrm{KOH}$ a $10 \%$. Se midieron apotecios, pelos del excípulo ectal, células del excípulo ectal, medular y componentes del himenio (paráfisis, ascas, ascosporas) utilizando un microscopio óptico K-7 (Zeiss, Jena, Alemania), los cuales se ilustraron en las descripciones y fotografiaron con una cámara D-7000 y un lente DX Micor 85 mm (Nikon, Tokio, Japón). El uso de los conceptos micológicos se basó en el Diccionario Ilustrado de Micología de Ulloa y Hanlin (2006). Los colores hacen referencia a la carta de color de Kornerup y Wanscher (1978).

\section{Resultados}

\section{Leotiomycetes}

\section{Cyttariales}

\section{Cordieritidaceae}

Unguiculariopsis ravenelii (Berk. \& M.A. Curtis) W.Y. Zhuang \& Korf, Mycotaxon 29: 395. 1987. Figs. 1-3.

= Peziza ravenelii Berk. \& M.A. Curtis, Grevillea 3(28): 152. 1875.

三 Cenangium ravenelii (Berk. \& M.A. Curtis) Sacc., Syll. Fung. (Abellini) 8: 568. 1889

= Encoeliella ravenelii (Berk. \& M.A. Curtis) Höhn., Sber. Akad. Wiss. Wien, Math.-naturw. Klasse., Abt. 1 119: 619. 1910

= Pithyella hamata Chenant., Bull. Soc. Mycol. Fr. 34: 39. 1918

= Unguiculariopsis ravenelii subsp. hamata (Chenant.) W.Y. Zhuang, Mycotaxon 32(1): 53. 1988

Apotecios 0.8-1 mm de diámetro, discoides a cupuliformes, subestipitados; margen enrollado a lobulado, himenio color naranja brillante (6A5) a naranja rojizo (7A7) cuando fresco, marrón vináceo (8D8) cuando seco, parte externa color marrón grisáceo (7C2), textura pruinosa por la presencia de pelos color hialino en $\mathrm{KOH}$ a $10 \%$, se desarrollan del excípulo ectal de 20-27 × 2.4-3.2 $\mu \mathrm{m}$, forma de gancho con el ápice curvado, paredes delgadas y lisas, amiloides en reactivo de Melzer; excípulo ectal 40-60 $\mu \mathrm{m}$ 


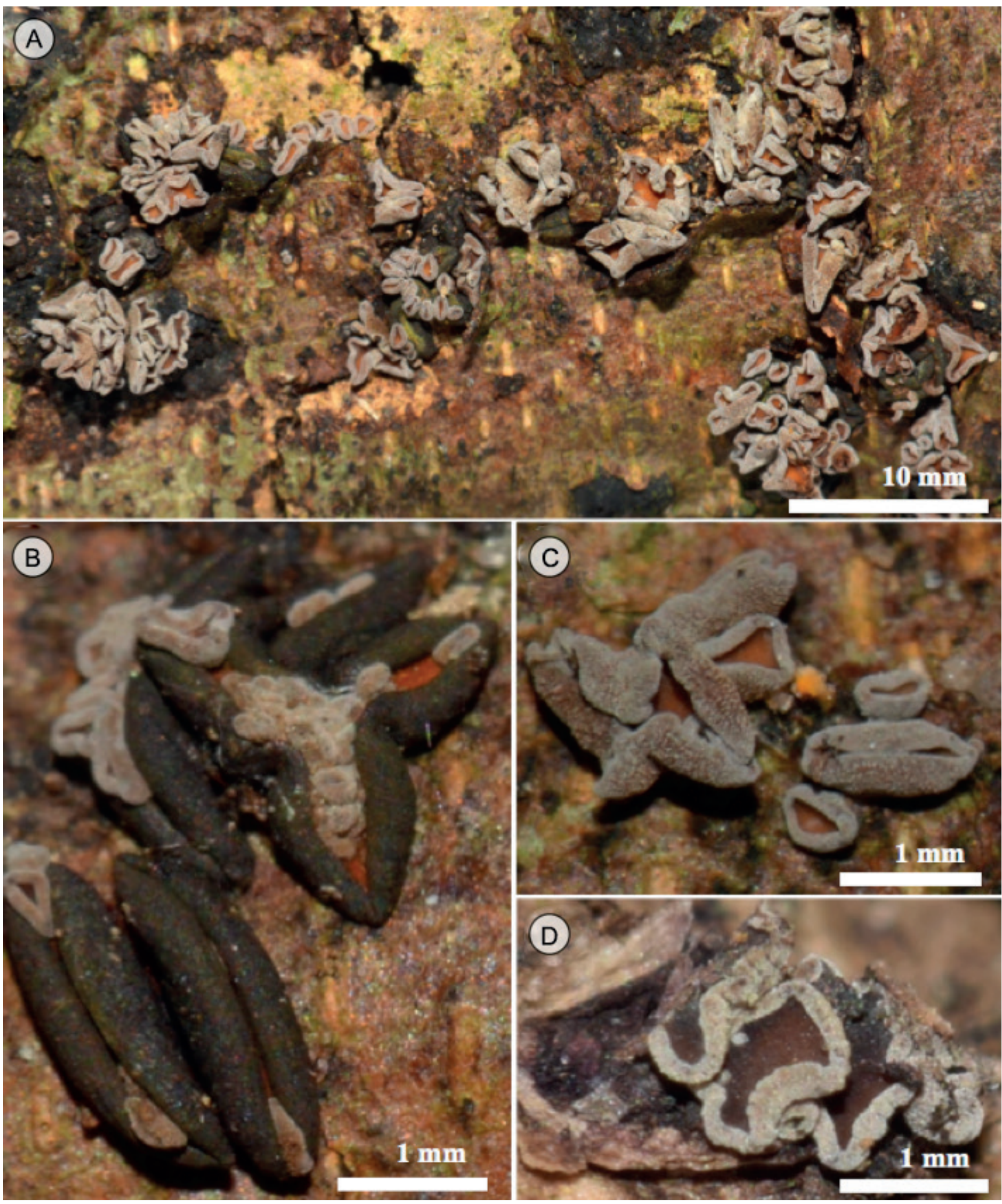

Figura 1: Unguiculariopsis ravenelii (Berk. \& M.A. Curtis) W.Y. Zhuang \& Korf. A. apotecios gregarios; B. apotecios creciendo sobre histerotecios de Rhytidhysteron rufulum (Spreng.) Speg.; C. apotecios frescos; D. apotecios deshidratados. 


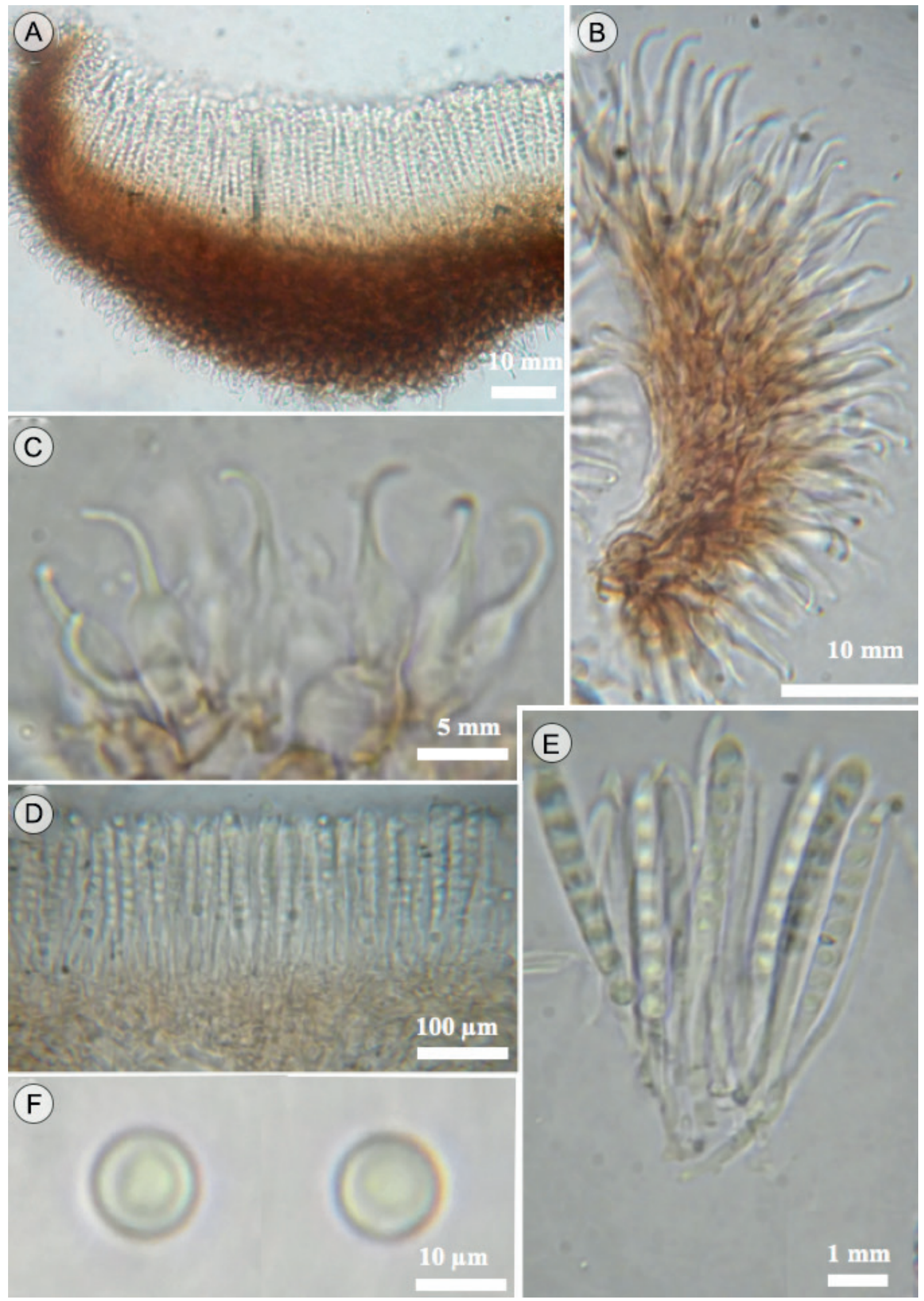

Figura 2: Unguiculariopsis ravenelii (Berk. \& M.A. Curtis) W.Y. Zhuang \& Korf. A. corte transversal de apotecio; B-C. pelos del excípulo ectal; D. himenio; E. ascas y paráfisis; F. ascosporas. 


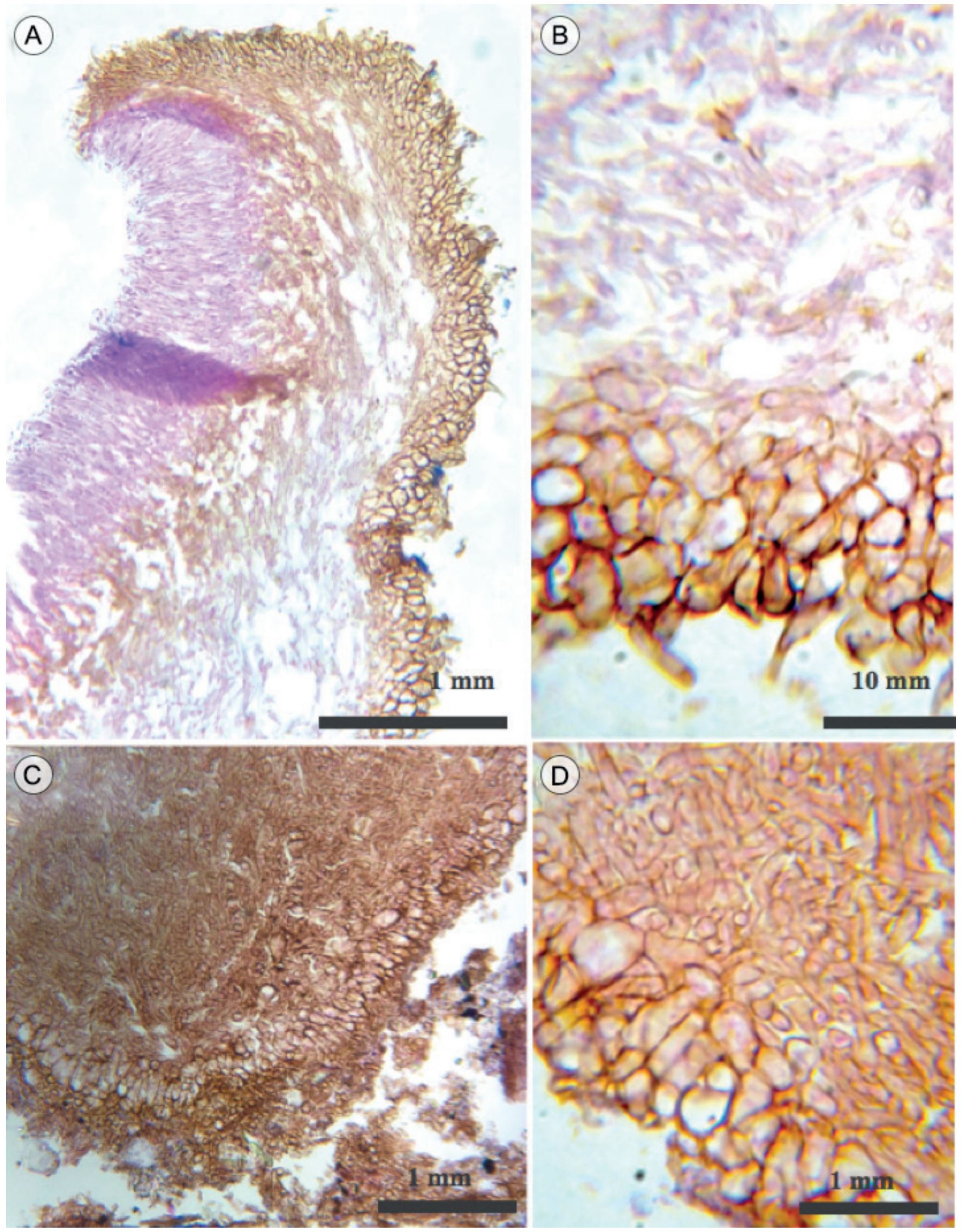

Figura 3: Unguiculariopsis ravenelii (Berk. \& M.A. Curtis) W.Y. Zhuang \& Korf. A. corte transversal de apotecio; B. excípulo medular, ectal y pelos; C. excípulo medular del subestípite; D. excípulo ectal del margen. 
de grosor, células 5-15 $\mu \mathrm{m}$ de diámetro, textura angularis, color marrón (7E8) a marrón rójizo (8E8); excípulo medular 50-120 $\mu \mathrm{m}$ de grosor, adelgazándose hacia el margen, hifas 4-6 $\mu \mathrm{m}$ de diámetro, textura intricada, color amarillento; subhimenio 53.2-60.8 $\mu \mathrm{m}$ de grosor; paráfisis 1.6-2.4 $\mu \mathrm{m}$ de ancho, filiformes, un septo en la base; ascas 40-50 × 4.45.4(-6) $\mu \mathrm{m}$, cilíndricas, hialinas, octospóricas, uniseriadas; ascosporas 3.2-4 $\mu \mathrm{m}$ de diámetro, globosas, hialinas, con gútulas, paredes gruesas y lisas.

Hábito y hábitat: fungícola, crece gregario sobre Rhytidhysteron rufulum, en México en bosque tropical caducifolio desde el nivel del mar hasta $500 \mathrm{~m}$.

Distribución: Boyacá y Santa Martha en Colombia, Carolina del Norte, Carolina del Sur y Florida en los Estados Unidos de América, Jamaica, Tingo María en Perú, Bolivar y Amazonas en Venezuela (Zhuang, 1988). Se cita por primera vez para México.

Material estudiado: MÉXICO. Colima, municipio Manzanillo, Humedales, $4 \mathrm{~km}$ al $\mathrm{N}$ de Manzanillo, 1907'49.11"N, 104¹7'27.489"O, 12.VII.2016, R. Valenzuela 16708 (ENCB), 16750 (ENCB). Jalisco, municipio La Huerta, vereda Búho, Reserva de la Biósfera Chamela-Cuixmala, 19²9'34"N, $105^{\circ} 02^{\prime} 38.26^{\prime \prime O}$, 15.IX.2018, S. Bautista-Hernández 926 (ENCB); loc. cit., 23.IX.2012, T. Raymundo 4449 (ENCB). Nayarit, municipio San Blas, km 27 de la carretera Tepic - San Blas, $21^{\circ} 31^{\prime} 45^{\prime \prime} \mathrm{N}, 105^{\circ} 09^{\prime} 48^{\prime \prime O}$; 25.X.2018, T. Raymundo 7963 (ENCB). Oaxaca, distrito Juquila, municipio San Pedro Tututepec, Parque Nacional Lagunas de Chacahua, $15^{\circ} 97^{\prime} 66^{\prime \prime} \mathrm{N}$, 97²13'67"O, 2.XI.2013, R. Valenzuela 15339 (ENCB), 16750 (ENCB); loc. cit., 2.XI.2013, T. Raymundo 5199 (ENCB). Puebla, municipio Honey, Cascadas Arcoíris, 20¹6'25.52"N, 98¹2'59.28"O, 25.XI.2017, M. Sánchez 1102a (ENCB). Sonora, municipio Álamos, Área de Protección de Flora y Fauna Sierra de Álamos-Río Cuchujaqui, rancho La Sierrita, 2659'01"N, $108^{\circ} 56^{\prime} 23^{\prime \prime O}$, 15.X.2013, R. Valenzuela 15224 (ENCB). Tabasco, municipio Emiliano Zapata, Unidad de Manejo Ambiental Manatí, 2652'48"N, 10904'48"O, 19.VII.2015, E. EscuderoLeyva 484 (ENCB); loc. cit., 19.VII.2015, R. Valenzuela 15600 (ENCB). Tamaulipas, municipio Gómez Farías, La Florida, ejido El Nacimiento, Reserva de la Biósfera El Cielo, 2259'25.08"N,
9908'38.04"O, 26.VI.2018, A. Cobos-Villagrán 1932 (ENCB), 1949 (ENCB), 1950 (ENCB). Municipio Victoria, Cañón del Novillo, 2342'50.33"N, 9907'09.11"O, 08.X.2019, M. Sánchez 1720 (ENCB).

\section{Discusión}

Unguiculariopsis ravenelii se caracteriza por formar apotecios de hasta $1 \mathrm{~mm}$ de diámetro, con himenio anaranjado y excípulo marrón grisáceo de textura pruinosa formado por pelos 20-27 $\mu \mathrm{m}$ de longitud con forma de gancho, ápice curvado y paredes lisas; ascas cilíndricas, octospóricas, uniseriadas y ascosporas de 3.2-4 $\mu \mathrm{m}$, globosas, hialinas y de paredes lisas. Una especie similar es $U$. hysterigena (Berk. \& Broome) Korf; no obstante, se diferencian por que forman apotecios con himenio marrón vináceo en fresco y excípulo grisáceo de textura pruinosa formado por pelos ganchudos, con el ápice curvado, de 16-22 $\mu \mathrm{m}$ de longitud con paredes granulosas a verrugosas; ascas cilíndricas, octospóricas, uniseriadas y ascosporas de 3.3-4.6 $\mu \mathrm{m}$ de diámetro, globosas, hialinas y de paredes lisas. La primera crece sobre Rhytidhysteron rufulum en América, la segunda sobre $R$. hysterinum (Dufour) Samuels \& E. Müll. y se distribuye tanto en China como en Sri Lanka.

Unguiculariopsis ravenelii es una especie fungícola o micoparásita que forma abundantes apotecios durante la temporada de lluvia en los bosques tropicales caducifolios de México, debido a la relación específica con su hospedero. Su distribución está ligada a la de Rhytidhysteron rufulum, la cual tiene su máxima expresión en ecosistemas tropicales y xerófilos (Cobos-Villagrán, 2019). La mayoría de los especímenes se distribuyen en las planicies costeras del Pacífico y del Golfo (Fig. 4). El presente estudio es el primer registro del género en México.

\section{Contribución de autores}

TR y RV concibieron y diseñaron el estudio. TR, MMP, ACV y MS contribuyeron a la adquisición de datos importantes para el trabajo. Las fotos de las figuras fueron tomadas por RV. TR escribió el manuscrito con la ayuda de RV. Todos los autores contribuyeron a la discusión, revisión y aprobación del manuscrito final. 


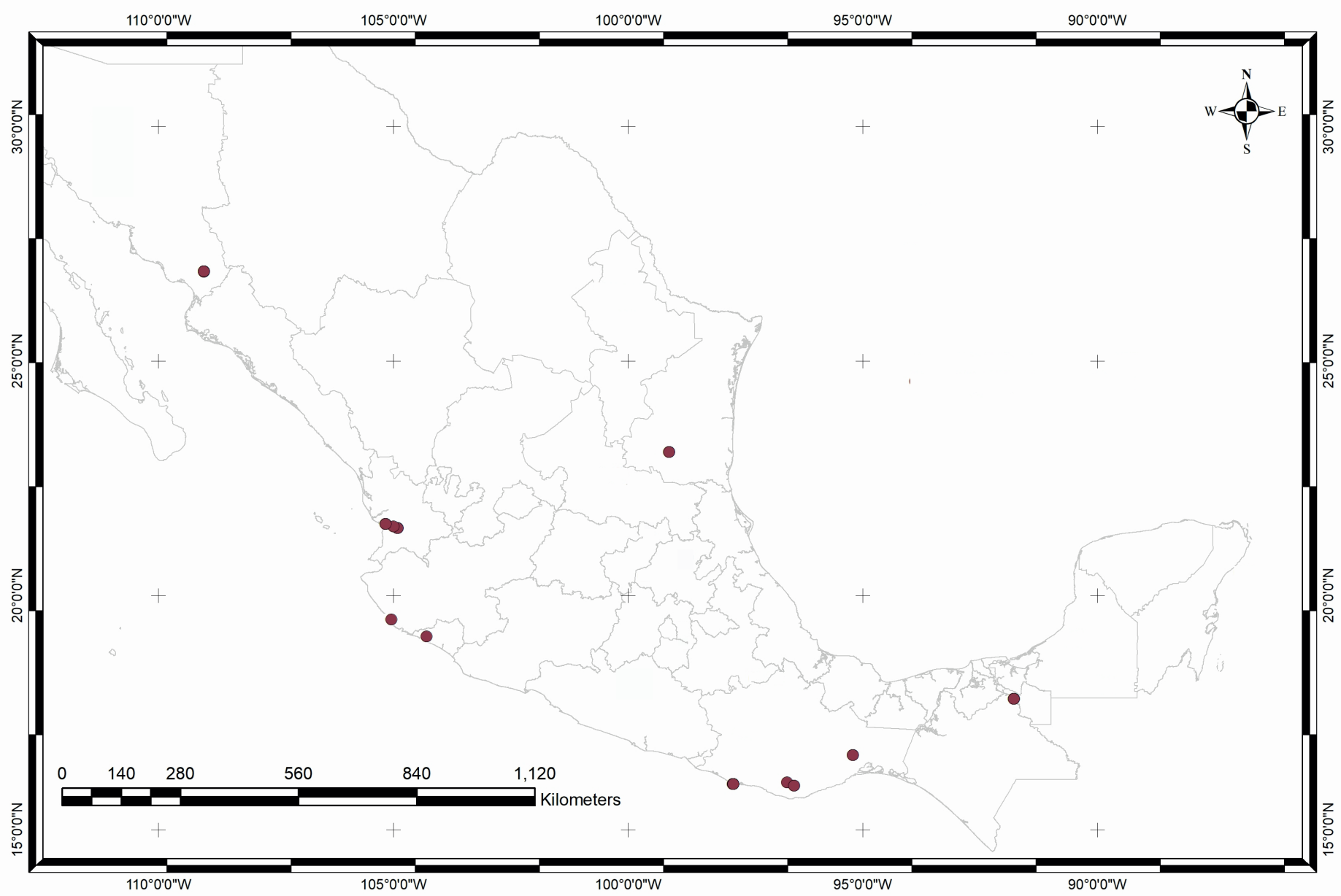

Figura 4: Ubicación de Unguiculariopsis ravenelii (Berk. \& M.A. Curtis) W.Y. Zhuang \& Korf en México.

\section{Financiamiento}

Este estudio fue apoyado por el Consejo Nacional de Ciencia y Tecnología (CONACYT) mediante el proyecto 252934 y por el Instituto Politécnico Nacional (IPN), a través de la Secretaria de Investigación y Posgrado (SIP) con los proyectos 20200248 y 20200956 . RV agradece, además, a la Comisión de Operación y Fomento de las Actividades Académicas (COFAA) del IPN y a la Secretaria de Posgrado e Investigación de la misma institución por el apoyo recibido mediante las becas de exclusividad (SIBE y EDI) para realizar sus investigaciones.

\section{Agradecimientos}

RV y TR agradecen a las autoridades de la ENCB, IPN, las facilidades otorgadas para realizar este estudio, en especial a los representantes de las Áreas Naturales Protegidas.

\section{Literatura citada}

Cobos-Villagrán, A. 2019. Sistemática del género Rhytidhysteron Speg. (Dothideomycetes: Ascomycota) en México. Tesis de maestría. Instituto Politécnico Nacional. Cd. Mx., México. $109 \mathrm{pp}$.

Ekanayaka, A. H., K. D. Hyde, E. Gentekaki, E. H. C. McKenzie, Q. Zhao, T. S. Bulgakov y E. Camporesi. 2019. Preliminary classification of Leotiomycetes. Mycosphere 10(1): 310489. DOI: https://doi.org/10.5943/mycosphere/10/1/7

GBIF. 2020. Global Biodiversity Information Facility. https://www. gbif.org/es/species/3487597 (consultado enero de 2020).

Hairaud, M. 2014. Contribution à la connaissance de Unguiculariopsis ravenelli subsp. hamata (Chenant.) W.Y. Zhuang. Ascomycete.org 6(5): 109-112. DOI: https://doi. org/10.25664/art-0112

Hawksworth, D. L. 1993. The tropical fungal biota: census, pertinence prophylaxis and prognosis. In: Isaac, S., J. C. 
Frankland, R. Watling y A. J. S. Whalley (eds.). Aspects of Tropical Mycology. Cambridge University Press. Cambridge, UK. Pp. 265-293.

Index Fungorum. 2020. Index Fungorum base de datos. http:// www.indexfungorum.org/names/Names.asp (consultado enero de 2020).

Korf, R. P. 1971. Some new Discomycete names. Phytopatologia 21(4): 201-207.

Kornerup, A. y J. H. Wanscher. 1978. Methuen Handbook of Colour. 3a ed. Eyre Methuen. London, UK. 252 pp.
Ulloa, M. y R. T. Hanlin. 2006. Nuevo Diccionario Ilustrado de Micología. American Phytopathological Society. St. Paul, USA. 672 pp.

Zhuang, W.-Y. 1988. A monograph of the genus Unguiculariopsis (Leotiaceae, Encoelioideae). Mycotaxon 32: 1-83.

Zhuang, W.-Y. 2000. Two new species of Unguiculariopsis (Helotiaceae, Encoelioideae) from China. Mycological Research 104(4): 507-509. DOI: https://doi.org/10.1017/ S0953756299001902 